\title{
Focus in Honor of David E. Clemmer, Recipient of the 2006 Biemann Medal
}

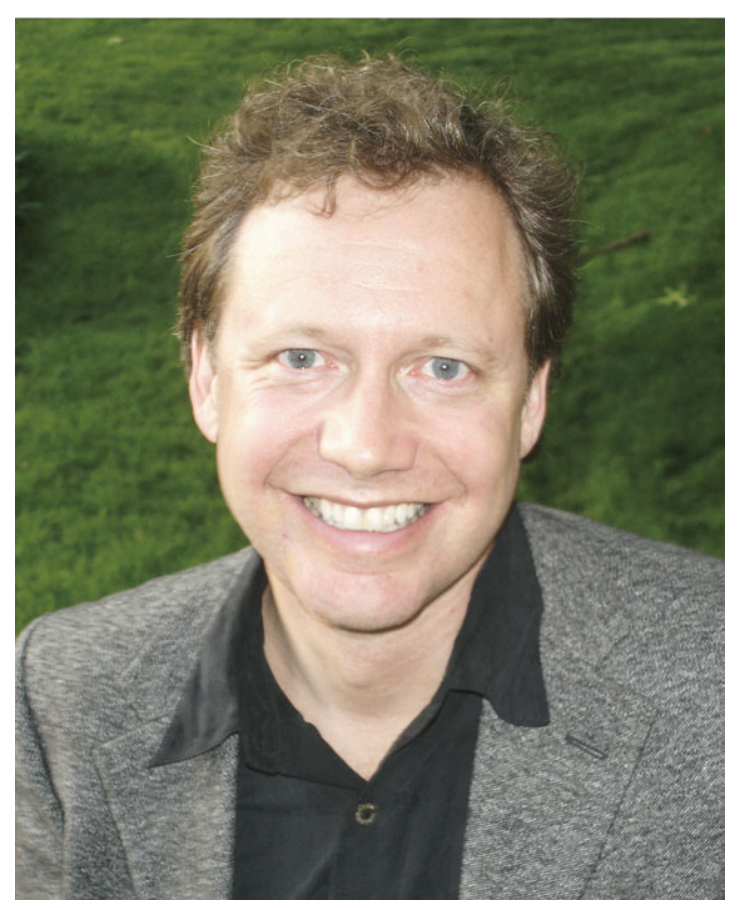

$\mathrm{T}$ The 2006 Biemann Medal was presented to Professor David E. Clemmer at the 54th ASMS Conference for his pioneering contributions to the integration of ion mobility separations with a variety of mass spectrometry technologies. This honor, as for so many in his young career, reflects David's incredible productivity, creativity, and impact on the field of mass spectrometry.

David received his Ph.D. in Physical Chemistry in 1992 from the University of Utah under the direction of Peter Armentrout. He served as a JSPS Postdoctoral Fellow at the Himeji Institute of Technology in Japan, and then as a Postdoctoral Associate at Northwestern University with Martin Jarrold. David joined the faculty of the Department of Chemistry at Indiana University in 1995, where he has risen to the rank of Full Professor and appointed to the Robert and Marjorie Mann Chair of Chemistry.

David has been exceptionally active in multiple scientific dimensions. He has published more than 125 refereed papers, presented about 150 lectures, mentored 20 graduate students, and holds four patents. He has garnered research support from NIH, NSF, ACS-PRF, the Sloan Foundation, the Dreyfus Foundation, Eli Lilly, Indiana University, and the ASMS. He is scientific cofounder of Beyond Genomics (a systems biology company aimed at understanding the molecular origins of diseased states) and founder of Predictive Physiology and Medicine (a biotechnology company aimed at providing detailed analytical measurements for personalized medicine). David has served the scientific community through many professional activities, including membership on the editorial boards of Analytical and Bioanalytical Chemistry, Analyst, Current Analytical Chemistry, The Protein Journal, and the International Journal of Mass Spectrometry. David has been honored by numerous prestigious awards, including the ACS Findeis Young Investigator Award, the TR-100 Research Innovation Award, the Phi Lambda Upsilon National Fresenius Award, and the Pittcon Achievement Award. He was named a member of Popular Science's 10 Most Brilliant list and selected as a Fellow of the Royal Society of Chemistry.

The editors of the Journal of the American Society for Mass Spectrometry are pleased to dedicate this Special Focus Issue on "From Mobilities to Proteomes" to David Clemmer. This issue highlights recent work from David's group as well as from eight other leading laboratories (it is unfortunate that the long list of willing contributors could not be accommodated in this issue). The first article, by Herbert Hill of Washington State University, demonstrates that electrospray ionization-ambient pressure ion mobility spectrometry/time-of-flight mass spectrometry is a rapid and effective analytical technique for the separation of carbohydrate isomers. The contribution by Richard Smith of the Pacific Northwest National Laboratories describes the development of a higher resolution ESI-IMSTOFMS instrument by utilizing electrodynamic ion funnels and elevated drift gas pressures. The article by Michael Bowers of the University of California, Santa Barbara explores the B-DNA helix stability in a solventfree environment through a combination of circular dichroism, ESI-MS, ion mobility, and molecular dynamics studies. The paper of Peter Armentrout of the University of Utah describes the development of an ion mobility source for guided ion beam experiments to study electronically excited states of atomic transition-metal cations. Joseph Loo of the University of California, Los Angeles demonstrates the application of electrospray ionization gas-phase electrophoretic mobility molecular analysis to evaluate the size of large proteins and protein complexes. The contribution from Evan Williams of the University of California, Berkeley describes studies of hydrated divalent clusters that are used as nanocalorimeters to measure the internal energy deposited upon electron capture dissociation. David Russell of Texas A \& M University presents a database of collision cross-sections of singly charged pep- 
tide ions, determined by a combination of MALDI, IMS, and TOF-MS. The article by Martin Jarrold of Indiana University describes studies of folding and unfolding of helix-turn-helix motifs in the gas phase utilizing ion mobility measurements and molecular dynamics simulations. In the final paper, David Clemmer describes mapping the human plasma proteome by SCX-LC-IMS-MS. This collection of papers shows clearly that "Ion Mobility" has come of age not only as a uniquely valuable new method for characterizing gaseous molecules, but that it can also dramatically improve specificity for routine qualitative and quantitative analysis.

David's remarkable accomplishments result from longer term research of truly exceptional creativity and breadth. As long-time admirers of David's career, we note that his earlier research involved pioneering fundamental physical chemistry. With Peter Armentrout he studied gaseous metal ion reactions with molecules, while his postdoctorate in Japan investigated excitedstate metal atom reactions with gaseous molecules. His postdoctorate with Martin Jarrold on metallofullerenes could appear similar, but, most importantly, this work introduced him to gaseous ion mobilities. David saw the tremendous potential for fundamental research and analytical applications, and his research program has taken off from there. The sampling of high quality papers and diverse topics in this issue is a very special tribute to his vision. Ion mobility is now an established, widely recognized field, and we mass spectrometrists selfishly claim it as one of our own.

David's own research first extended the pioneering studies of his predecessors Mike Bowers and Martin Jarrold that showed ion mobility to be a powerful new tool for characterizing the conformations of gaseous ions. This new field, proposed as of special importance for insights into protein folding and enzymatic specificity, inspired a variety of studies, but often of disparate conclusions. David's thorough studies of small proteins such as ubiquitin established a fundamental relationship between collision cross section values and postulated structures. Not only did different charge states have different structures, but he found conclusive evi- dence that a single charge state could exist in multiple conformations. This is in direct contrast to the singularity of a protein's native conformation in solution, even with varying $\mathrm{pH}$.

Revolutionary instrumental methods key to the "Analytical Revolution" in science are the "hyphenated techniques" such as GC/MS and 2D NMR. David recognized and implemented such a combination of ion mobility with mass spectrometry (IMS-MS), and this truly has added another promising dimension to MS. Two peptides can have the same exact mass value, even with modern high-resolution MS capabilities; further, with the best LC conditions, these peptides still may not be separated. Clemmer's unique LC-IMS-MS, as described in this issue, provides even more specific information for the on-line analyses of complex peptide mixtures, a highly promising, powerful new tool for proteomics.

It is especially appropriate that efficient, high sensitivity peptide characterization is an important accomplishment of the Biemann Medalist. At David's age, MIT's Professor Klaus Biemann had already proposed and demonstrated the unique capabilities of mass spectrometry in sequencing peptides in amounts far smaller than seemed conceivable by the analytical standards of the day. The Journal of the American Society for Mass Spectrometry is privileged to present this issue to highlight David Clemmer's similar accomplishments. Ion mobility spectrometry is now a field of growing importance, and we predict that it will demand an increasing proportion of this journal's pages.

Veronica M. Bierbaum Department of Chemistry and Biochemistry University of Colorado

Boulder, Colorado, USA

Fred W. McLafferty Department of Chemistry and Chemical Biology Cornell University

Ithaca, New York, USA 